\title{
Evaluación positiva de medicamentos: 1.er trimestre 2013
}

\author{
Sánchez López P. ${ }^{1}$, Cabanes Mariscal MA. ${ }^{2}$, García Luque A. ${ }^{3}$, Puerro Vicente M. ${ }^{4}$
}

Sanid. mil. 2013; 69 (2): 98-101; ISSN: 1887-8571

\section{RESUMEN}

Se reseñan los medicamentos ya evaluados por la Agencia Española de Medicamentos y Productos Sanitarios hechos públicos en el primer trimestre de 2013, y considerados de mayor interés para el profesional sanitario en el ámbito hospitalario. Se trata de opiniones técnicas positivas que son previas a la autorización y puesta en el mercado del medicamento.

PALABRAS CLAVE: Bosutinib, Bosulif, Ocriplasmina, Jetrea, Teriflunomida, Aubagio, Inmunoglogulina Humana Normal, HyQvia, Ponatinib, Iclusig, Elvitegravir/ Cobicistat/ Emtricitabina/ Tenofovir Disoproxil, Stribild, Dimetilfumarato, Tecfidera.

\section{Positive assessment of drugs: $1^{\text {st }}$ quarter 2013}

SUMMARY: The drugs assessed by the Spanish Agency for Medicines and Health Products made public in the first quarter of 2013, and considered of interest in hospital healthcare professional, are reviewed. These are positive technical reports prior to the authorization and placing on the market of the product.

KEY WORDS: Bosutinib, Bosulif, Ocriplasmina, Jetrea, Teriflunomida, Aubagio, Inmunoglogulina Humana Normal, HyQvia, Ponatinib, Iclusig, Elvitegravir/ Cobicistat/ Emtricitabina/ Tenofovir Disoproxil, Stribild, Dimetilfumarato, Tecfidera.

\section{BOSUTINIB (Bosulif®) $)^{1-3}$}

El principio activo es bosutinib, un inhibidor de proteína quinasa, que actúa inhibiendo la quinasa Bcr-Abl defectuosa que promueve la leucemia mieloide crónica (LMC). Esta indicado para el tratamiento de pacientes adultos con leucemia mieloide crónica y cromosoma Philadelphia positivo que se encuentren en fase crónica, acelerada y blástica que hayan sido previamente tratados con uno o más inhibidores de tirosina quinasa y para los que imatinib, nilotinib y dasatinib no se consideran opciones de tratamiento adecuadas.

El Comité Europeo de Evaluación de Medicamentos (CHMP) ha recomendado de manera condicional la autorización de comercialización de Bosulif $®$, al satisfacer una necesidad médica no cubierta, en la medida en que, el beneficio para la salud pública de su inmediata disponibilidad es superior al riesgo inherente de que todavía se requieran datos adicionales.

En los ensayos clínicos, las tasas de respuesta hematológica o citogenética fueron mayores para bosutinib que para su comparador (imatinib) en la población estudiada.

El ensayo clínico pivotal fue un ensayo abierto, no controlado, en fase I/II que evaluó la eficacia y seguridad de bosutinib en 570 pacientes con cromosoma Philadelphia positivo que habían sido previamente tratados con, al menos, un inhibidor de la ti-

${ }^{1}$ Cte. Farmacéutico. Servicio de Farmacia Hospitalaria

${ }^{2}$ Tcol. Farmacéutico. Servicio de Farmacia Hospitalaria

${ }^{3}$ Cte. Médico. Servicio de Farmacología Clínica.

${ }^{4}$ Tcol. Médico. Servicio de Farmacología Clínica.

Hospital Central de la Defensa Gómez Ulla. Madrid. España.

Dirección para correspondencia: Servicio de Farmacia Hospitalaria. Hospital Central de la Defensa Gómez Ulla. 28047 Madrid. España.mgonalf@oc.mde.es

$\overline{\text { Recibido: } 19}$ de abril de 2013

Aceptado: 25 de abril de 2013 rosin quinasa. Hubo 52 pacientes en los cuales los resultados se consideraron como una evidencia principal, dado que no eran candidatos a tratamiento con otros inhibidores de protein quinasa (bien por resistencia o bien por intolerancia), y por tanto tenían una necesidad médica no cubierta por ningún otro fármaco. Entre estos 52 pacientes, 36 tenían enfermedad en fase crónica y 16 tenían enfermedad en fase blástica o acelerada. La variable principal de eficacia del estudio fue el número de pacientes que lograban al menos una «respuesta citogenética mayor» (cuando la proporción de glóbulos blancos con cromosoma Philadelphia caía por debajo del 35\%) después de seis meses de tratamiento con bosutinib. Otras variables de eficacia secundarias incluían la «respuesta hematológica» (recuento normal de globulos blancos). Bosutinib demostró ser eficaz en pacientes sin sus necesidades médicas cubiertas, 18 de los 36 pacientes con enfermedad en fase crónica tuvieron una «respuesta citogenética mayor», mientras que 7 de los 16 pacientes con enfermedad en fase blástica o acelerada obtuvieron respuesta suficiente en base a otras variables de eficacia.

Las reacciones adversas observadas con más frecuencia durante el desarrollo clínico fueron: diarrea, náuseas, trombocitopenia, vómitos, dolor abdominal, rash, anemia, pirexia y un aumento de alanina aminotranferasa.

Se recomienda su prescripción por médicos con experiencia en el diagnóstico y tratamiento de la LMC. Bosulif fue designado como medicamento huérfano el 4 de agosto de 2010.

\section{DIMETILFUMARATO ( Tecfidera $\left.{ }^{\circledR}\right)^{4-6}$}

El principio activo es el dimetilfumarato, que actúa principalmente desencadenando la activación de la vía transcripcional Nrf2. 


\section{Evaluación positiva de medicamentos: 1. er $^{\text {trimestre }} 2013$}

Indicación aprobada: Tratamiento de pacientes adultos con esclerosis múltiple remitente recidivante.

La vía Nrf2/ARE es una diana emergente para una nueva estrategia terapéutica frente a la ELA y la EP al interferir en la inflamación y el estrés oxidativo. ${ }^{7}$

En los ensayos clínicos, el dimetilfumarato ha mostrado reducir la tasa de recaídas en pacientes con esclerosis múltiple remitente recidivante. La aprobación se basa en diversos estudios que han demostrado clínicamente que dimetilfumarato reduce de manera significativa los brotes y el desarrollo de lesiones cerebrales y retrasa la progresión de la discapacidad a lo largo del tiempo, demostrando un perfil de seguridad y tolerabilidad favorable. En concreto, la aprobación se basa en los datos obtenidos de dos estudios globales de fase III: 'Define' y 'Confirm', en los que participaron más de 2.600 pacientes. Asimismo, en el estudio de extensión en curso, 'Endorse', se ha hecho un seguimiento de algunos pacientes durante más de cuatro años. En estos ensayos clínicos, los pacientes tratados con dimetilfumarato presentaron menor actividad de la enfermedad en comparación con pacientes tratados con placebo, tanto si estaban en etapas iniciales de la enfermedad, como si la enfermedad estaba en estadios más avanzados.

Las reacciones adversas observadas con más frecuencia durante el desarrollo clínico fueron: sofocos y problemas gastrointestinales como diarrea, náuseas y dolor abdominal.

Se recomienda que el tratamiento con dimetilfumarato sea iniciado y supervisado por médicos con experiencia en el tratamiento de la esclerosis múltiple.

\section{ELVITEGRAVIR/ COBICISTAT/ EMTRICITABINA/ TENOFOVIR DISOPROXIL (Stribild $\left.{ }^{\circledR}\right)^{4,7}$}

Los principios activos son elvitegravir/ cobicistat/ emtricitabina/ tenofovir disoproxil, una combinación de antivirales para el tratamiento de infecciones por VIH. Elvitegravir es un inhibidor de la integrasa del VIH-1; cobicistat aumenta la exposición sistémica de elvitegravir; emtricitabina es un análogo de nucleósido de la citidina y tenofovir, es un análogo de nucleósido monofosfato (nucleótido) del monofosfato de adenosina.

La Indicación aprobada es el tratamiento de la infección por el virus de la inmunodeficiencia humana tipo 1 (VIH-1) en pacientes adultos a partir de los 18 años que no han recibido tratamiento previo antirretroviral, o que estén infectados por el VIH-1 sin mutaciones conocidas asociadas a resistencias a cualquiera de los tres agentes antirretrovirales incluidos en Stribild $\AA$.

Actualmente en España disponemos de Raltegravir, otro agente inhibidor de la integrasa (nueva clase de antiretrovirales de reciente aprobación). Emtricitabina y tenofovir ya los teníamos en nuestro arsenal terapéutico en asociación (Trubada®).

En los ensayos clínicos Stribild $\AA$, ha mostrado reducir la carga viral a niveles indetectables ( $<50$ copias /ml de ARN de VIH-1). Uno de los dos estudios clínicos presentados comparó Stribild versus Atripla. Fue un estudio clínico fase 111 aleatorizado en el que participaron alrededor de 700 pacientes. El estudio demostró que Stribild y Atripla tuvieron efectos similares en términos de reducción de la carga viral en los pacientes. El 88\% de los pacientes que recibió Stribild logró supresión de la carga viral tras un promedio de 48 semanas de tratamiento, versus un $84 \%$ de los pacientes en el grupo Atripla. El segundo estudio clínico también se realizó en una muestra poblacional de cerca de 700 pacientes y comparó la respuesta de pacientes tratados con Stribild, versus la respuesta de pacientes que recibieron un régimen de Truvada más atazanavir / ritonavir. En este segundo estudio Stribild redujo la carga viral en $90 \%$ de los pacientes después de 4 semanas, mientras que el tratamiento Truvada-atazanavirritonavir redujo la carga viral en el $87 \%$ de los pacientes.

Las reacciones adversas observadas con más frecuencia durante el desarrollo clínico fueron: hipofosfatemia, dolor de cabeza, mareos, diarrea, vómitos, náuseas, erupción cutánea, astenia y elevación de creatina quinasa. Los tipos de reacciones adversas renales observados con Stribild $₫$ fueron consistentes con la experiencia previa con tenofovir disoproxil fumarato. Actualmente no existen datos suficientes para determinar si la administración concomitante de tenofovir disoproxil fumarato y cobicistat está asociada con un mayor riesgo de reacciones adversas renales en comparación con regímenes que incluyen tenofovir disoproxil fumarato sin cobicistat. Se recomienda su prescripción por médicos con experiencia en el tratamiento de la infección por VIH.

\section{INMUNOGLOGULINA HUMANA NORMAL $\left(\text { HyQvia }{ }^{\circledR}\right)^{4,8}$}

El principio activo es una inmunoglobulina humana normal que contiene principalmente inmunoglobulina $\mathrm{G}(\mathrm{IgG})$ con un amplio espectro de anticuerpos opsonizantes y neutralizantes contra agentes infecciosos.

Son dos las indicaciones aprobadas:

- Tratamiento de reposición en adultos ( $\geq 18$ años) con síndromes de inmunodeficiencia primaria como:

- Agammaglobulinemia congénita e hipogammaglobulinemia.

- Inmunodeficiencia variable común.

- Inmunodeficiencia combinada severa.

- Deficiencias de subclases de IgG con infecciones recurrentes.

- Tratamiento de reposición en adultos ( $\geq 18$ años) con mieloma o leucemia linfocítica crónica con hipogammaglobulinemia secundaria grave e infecciones recurrentes.

Actualmente disponemos ya en España de otra Inmunoglobulina humana inespecífica (s.c) Vivaglobin ${ }^{\circledR}$.

En los ensayos clínicos, HyQvia disminuyó el porcentaje de infecciones bacterianas agudas y graves por año. HyQvia se administra vía subcutánea cada 3 ó 4 semanas con una infusión inicial con hialuronidasa humana recombinante que facilita la dispersión y absorción de IG10\%.

Este nuevo medicamento, podría ofrecer a los pacientes la opción de administrar la terapia en casa, con una administración subcutánea cada tres o cuatro semanas, lo que potencialmente puede hacer que las reacciones adversas sistémicas sean menores en comparación con los tratamientos intravenosos.

Las reacciones adversas observadas con más frecuencia durante el desarrollo clínico fueron: reacciones locales (presente en el $20 \%$ de las infusiones mostrando malestar/dolor, eritema, hinchazón/edema y prurito), cefalea (3\%), fatiga ( $1 \%$ y fiebre $(1 \%)$. 
Se recomienda su prescripción por médicos con experiencia en el tratamiento de las inmunodeficiencias.

\section{OCRIPLASMINA (Jetrea $\left.{ }^{\circledR}\right)^{1,9}$}

El principio activo es ocriplasmina, que posee actividad proteolítica frente a componentes proteicos del cuerpo vítreo y de la interfaz vitreoretiniana (por ejemplo, laminina, fibronectina y colágeno) con el fin de disolver la matriz proteica responsable de la adhesión vitreomacular defectuosa. La fuerte unión de los componentes proteicos dentro del área macular de la interfaz vitreoretiniana contribuye a la tracción vitreomacular originando alteraciones visuales y /o agujeros maculares.

Ha sido aprobado para el tratamiento de la tracción vitreomacular en adultos, incluyendo cuando ésta está asociada a un agujero macular de un diámetro menor o igual a 400 micras. Este medicamento representa el primer tratamiento farmacológico ocular para esta dolencia. La adherencia vitreomacular (AVM), que incluye la tracción vitreomacular (TVM) y los agujeros maculares, afecta a más de 300.000 personas sólo en Europa, y en ambos casos son dolencias oculares incapacitantes, progresivas y relacionadas con la edad que pueden provocar distorsión visual, pérdida de agudeza visual y ceguera central. Hasta ahora estos pacientes sólo podían optar a una intervención quirúrgica en una etapa más tardía de la enfermedad.

En los ensayos clínicos de fase III han participado 652 pacientes, y en ellos ocriplasmina ha mostrado que libera la tracción entre el vítreo y la mácula gracias a su actividad proteolítica, con el potencial de prevenir daños mayores en la visión y la necesidad de vitrectomía en un pequeño grupo de pacientes. Después de 28 días, tras una única administración de ocriplasmina, se observó resolución de la adherencia vitreomacular en el 26'5\% de los pacientes frente al 10'1\% en el grupo con placebo ( $p<0$ '001). Esta diferencia estadísticamente significativa se mantuvo durante los seis meses de observación. En la mayoría de los pacientes que consiguieron una resolución de la adherencia vitreomacular tras la administración de ocriplasmina, esta se produjo en un plazo de siete días. Al final del periodo de observación de seis meses, los pacientes que necesitaron una vitrectomía fueron menos en el grupo tratado con ocriplasmina, que en el grupo con placebo $(17,7 \%$ frente al $26,6 \%$ respectivamente, $p=0,02)$.

Las reacciones adversas observadas con más frecuencia durante el desarrollo clínico fueron: cuerpos flotantes en el vítreo, dolor ocular y fotopsia, y hemorragia conjuntival relacionada con la inyección. Debe ser preparado y administrado por un oftlamólogo con experiencia en administración intravítrea.

\section{PONATINIB (Iclusig $\left.{ }^{\circledR}\right)^{4,10}$}

El principio activo es ponatinib, un inhibidor de la tirosinaquinasa Bcr-Abl.

La indicación aprobada es para pacientes adultos diagnosticados de:

- Leucemia mieloide crónica (LMC) en fase crónica, acelerada o blástica que sean resistentes a dasatinib o nilotinib; que sean intolerantes a dasatinib o nilotinib y para quie- nes no sea apropiado el tratamiento posterior con imatinib desde el punto de vista clínico; o tengan la mutación T315I.

- Leucemia linfoblástica aguda cromosoma Filadelfia positivo (LLA $\mathrm{Ph}+)$ que sean resistentes a dasatinib; que sean intolerantes a dasatinib y para quienes no sea apropiado el tratamiento posterior con imatinib desde el punto de vista clínico; o tengan la mutación T315I.

Tanto dasatinib, nilotinib como imatinib ya están autorizados en España para esas patologías oncológicas.

En los ensayos clínicos, ponatinib ha mostrado tasas de respuesta citogenética y hematológica en pacientes con LMC y LLA $\mathrm{Ph}+$, incluyendo pacientes con la mutación T315I. En el principal ensayo clínico para evaluar su eficacia participaron 449 pacientes con LMC y LLA Ph+. Los investigadores encontraron que había una reducción significativa en el número de células cancerosas con la mutación genética cromosoma Filadelfia, que lograron una respuesta citogenética mayor (RCM). Esta RCM se logró en el $70 \%$ de los pacientes con la mutación T315I y en $54 \%$ de todos los pacientes. Durante la fase blástica y LLA Ph+ encontraron que el $52 \%$ de los participantes en fase acelerada de LMC no experimentaron ninguna evidencia de leucemia (respuesta hematológica mayor, AMDH) durante una mediana de 9'5 meses. Un 31\% de los participantes con LMC en fase blástica experimentaron mayor respuesta hematológica durante un período medio de 47 meses. Y el 41\% de los participantes con LLA $\mathrm{Ph}+\mathrm{Mahr}$ durante una mediana de 3'2 meses.

Las reacciones adversas observadas con más frecuencia durante el desarrollo clínico fueron: descenso del recuento de plaquetas, rash, piel seca y dolor abdominal. Se recomienda su prescripción por médicos con experiencia en el diagnóstico y tratamiento de pacientes con leucemia. Fue designado como medicamento huérfano el 2 de febrero de 2010.

\section{TERIFLUNOMIDA (Aubagio $\left.{ }^{\circledR}\right)^{4,11}$}

El principio activo es teriflunomida, un inmunosupresor selectivo con propiedades antiinflamatorias. No se conoce con precisión el mecanismo de acción de teriflunomida, pero si se sabe que disminuye la proliferación de linfocitos mediante el bloqueo de la enzima mitocondrial dihidroorotato deshidrogenasa (DHO-DH).

La indicación aprobada por la EMA es el tratamiento de pacientes adultos con esclerosis múltiple remitente recidivante (recaídas con recuperación total o secuelas y déficit residual en la recuperación sin progresión).

Esta enfermedad crónica dispone, actualmente en España, como tratamiento de $1^{\mathrm{a}}$ línea de IFN-beta, acetato de glatirámero y azatioprina y como tratamiento de $2^{\mathrm{a}}$ línea mitoxantrona y natalizumab ( $1^{\mathrm{a}}$ en formas agresivas de inicio).

En los ensayos clínicos, teriflunomida ha mostrado reducir la tasa de recaídas en pacientes con esclerosis múltiple remitente recidivante. La aprobación se ha basado en los datos de eficacia del ensayo TEMSO (Teriflunomide Multiple Sclerosis Oral). TEMSO fue un estudio internacional de dos años de duración, aleatorizado, doble ciego, controlado con placebo que incluyó a 1.088 pacientes con formas recidivantes de EM en 126 centros en 21 países. Los participantes del ensayo tenían 


\section{Evaluación positiva de medicamentos: 1. er trimestre 2013}

entre 18-55 años de edad, con una Escala Expandida del Estado de Discapacidad (EDSS, siglas de su nombre en inglés) de 5'5 o menos, y habían tenido al menos una recaída durante el año anterior o por lo menos dos recaídas en los dos años anteriores. En este ensayo de fase III, $14 \mathrm{mg}$ de teriflunomida redujeron significativamente la tasa anual de recaídas $\left(\mathrm{p}=00^{\prime} 0005\right)$ y el tiempo hasta la progresión de la discapacidad $(\mathrm{p}=0$ '0279) a los dos años en comparación con placebo en pacientes con formas recurrentes de la esclerosis múltiple. Siete mg de teriflunomida redujeron significativamente la tasa anual de recaídas ( $p=0$ '0002) en el ensayo.

Las reacciones adversas observadas con más frecuencia durante el desarrollo clínico fueron: infecciones del tracto respiratorio superior, infecciones del tracto urinario, diarrea, náuseas, parestesias (entumecimiento y hormigueo), alopecia (pérdida de cabello) y aumento del nivel de la enzima hepática alanina aminotransferasa.

Se recomienda que el tratamiento con teriflunomida sea iniciado y supervisado por médicos con experiencia en el tratamiento de la esclerosis múltiple

\section{BIBLIOGRAFIA}

1. Agencia Española de Medicamentos y Productos Sanitarios. Informe mensual sobre Medicamentos de Uso Humano y Productos Sanitarios Marzo 2013. Disponible en: http://www.aemps.gob.es/informa/informeMensual/2013/enero/informe-medicamentos.htm\#p1(consultado 15/04/13)

2. Committee for Orphan Medicinal Products (COMP) EMA/327056/2013 16 June 2011 Disponible en: http://www.ema.europa.eu/docs/en_GB/document_library/Orphan_designation/2010/08/WC500095733.pdf (consultado $15 / 04 / 13)$
3. Committee for Medicinal Products for Human Use (CHMP) EMA/42251/2013 Disponible en: http://www.ema.europa.eu/ema/index. jsp?curl=pages/medicines/human/medicines/002373/human_med_001613. jsp\&mid=WC0b01ac058001d124 (consultado 16/04/13)

4. Agencia Española de Medicamentos y Productos Sanitarios. Informe mensual sobre Medicamentos de Uso Humano y Productos Sanitarios Marzo 2013. Disponible en: http://www.aemps.gob.es/informa/informeMensual/2013/marzo/docs/informe-mensual_marzo-2013.pdf (consultado 15/03/2013).

5. Committee for Medicinal Products for Human Use (CHMP) EMA/167897/2013 21 March 2013 Disponible en: http://www.ema.europa. eu/docs/en_GB/document_library/Summary_of_opinion_-_Initial_authorisation/human/002601/WC500140695.pdf (consultado 15/03/2013).

6. R.A. Linker, D.H.Lee, S. Ryan, A.M. van Dam, R. Conrad et al. Fumaric acid esters exerts neuroprotective effects in neuroinflammation via activation of the Nrf2 antioxidant pathway Brain 134,3:678-692 2011. Disponible en http:// brain.oxfordjournals.org/content/134/3/678.short. (consultado 15/03/2013).

7. Committee for medicinal products for human use (CHMP) EMA/ CHMP/158160/2013 21 March 2013 http://www.ema.europa.eu/docs/en_ GB/document_library/Summary_of_opinion_-_Initial_authorisation/human/002574/WC500140660.pdf (consultado 15/03/2013).

8. Committee for medicinal products for human use (CHMP). EMA/ CHMP/38042/2013 21 March 2013 http://www.ema.europa.eu/docs/en_GB/ document_library/Summary_of_opinion___Initial_authorisation/human/002491/WC500140692.pdf (consultado 15/03/2013).

9. Committee for Medicinal Products for Human Use (CHMP) EMA/5734/2013 17 January 2013 Disponible en: http://www.ema.europa.eu/ema/index. jsp?curl=pages/medicines/human/medicines/002381/smops/Positive/human_smop_000466.jsp\&mid=WC0b01ac058001d127\&source $=$ homeMedSearch\&category=human (consultado 16/04/2013)

10. Committee for medicinal products for human use (CHMP). EMA/ CHMP/178415/2013 21 March 2013 Disponible en: http://www.ema.europa. eu/docs/en_GB/document_library/Summary_of_opinion_-_Initial_authorisation/human/002695/WC500140659.pdf (consultado 15/03/2013).

11. Committee for Medicinal Products for Human Use (CHMP) EMA/167896/2013 21 March 2013 Disponible en: http://www.ema.europa. eu/docs/en_GB/document_library/Summary_of_opinion_-_Initial_authorisation/human/002514/WC500140690.pdf (consultado 15/03/2013). 